\title{
Desenlaces clínicos de una cohorte de pacientes con cáncer de mama localmente avanzado manejadas con terapia hormonal adyuvante en el Instituto Nacional de Cancerología (INC)
}

Clinical results of patients with locally advanced breast cancer managed with neoadjuvant chemotherapy and received adjuvant hormonal therapy at the National Cancer Institute (INC)

\footnotetext{
- Diego Lizarazo', Jesús O. Sánchez², Sandra Díaz ${ }^{3}$

MD, Fellow Oncología Clínica, Instituto Nacional de Cancerología (Bogotá, D.C.).

MD, MSc Oncólogo Clínico, magister en Epidemiología Clínica, Instituto Nacional de Cancerología (Bogotá, D.C.)

${ }^{3}$ Cirujana, especialista en Cirugía de Seno y Tejidos Blandos, Instituto Nacional de Cancerología (Bogotá, D.C.).
}

\section{Resumen}

Introducción: para Colombia, según datos de Globocan 2018, el cáncer de mama es el tipo de cáncer más común en las mujeres con 13.380 casos representando el $24,8 \%$ de todos los casos.

Objetivo: describir la supervivencia libre de enfermedad y la supervivencia global de pacientes con cáncer de mama localmente avanzado, manejadas con quimioterapia neoadyuvante en el Instituto Nacional de Cancerología (INC) (Bogorá, Colombia) durante los años 2012 a 2014 y que además recibieron tratamiento adyuvante hormonal.

Métodos: estudio observacional descriptivo retrospectivo. La muestra fue por conveniencia de pacientes con cáncer de mama localmente avanzado que fueron manejadas con terapia hormonal adyuvante.

Resultados: se incluyeron 77 pacientes. La mediana de seguimiento fue de 4,6 años. La media de edad fue 53 años. La mayoría de las pacientes fueron clasificadas en estadio clínico IIIB $(51,9 \% n=40)$ y el subtipo biológico tumoral más común fue luminal B HER2 negativo $45,5 \%(n=35)$. La frecuencia de recurrencia (local, regional y sistémica) fue del 16,9\% ( $n=13)$. El tratamiento hormonal adyuvante más utilizado fue la terapia switch en el $40,3 \%(n=31)$ de los casos. No se encontraron diferencias en supervivencia libre de enfermedad y global de acuerdo con el subtipo tumoral biológico y el tipo de tratamiento hormonal adyuvante recibido. En cuanto al perfil de seguridad, el tratamiento administrado fue bastante seguro en cuanto a toxicidad, más frecuentes fueron las artralgias en el $11,7 \%(n=9)$ y la osteoporosis en el $10,4 \%(n=8)$.

Conclusiones: la mediana de supervivencia libre de enfermedad y global fue de 30 meses y 45,2 meses, respectivamente; no se documentaron diferencias entre los diferentes subtipos; se observó que los tumores luminal $A$, al igual que los pacientes que recibieron terapia de manipulación hormonal con esquema switch, tenían una menor frecuencia de recurrencia y mejor supervivencia global.

Palabras clave (DeCS): neoplasias de la mama, inhibidores de la aromatasa, tamoxifeno, mortalidad, supervivencia.

\begin{abstract}
Background: For Colombia, according to Globocan 2018 data, breast cancer is the most common type of cancer in women with 13.380 cases representing $24.8 \%$ of all cases.

Objective: Describe the disease-free survival and overall survival of patients with locally advanced breast cancer managed with neoadjuvant chemotherapy at the National Cancer Institute (INC) (Bogotá, Colombia) during 2012 to 2014 and received adjuvant hormonal therapy as part of their treatment.

Methods: This is an observational descriptive retrospective study. The sample was for convenience of patients with locally advanced breast cancer who were managed with adjuvant hormonal therapy.

Results: 77 patients were included. The median follow-up was 4.6 years. The average age was 53 years. The most of patients were classified in clinical stage IIIB $(51.9 \%, n=40)$ and the most common tumor biological subtype was luminal B
\end{abstract}


HER2 negative $45.5 \%(n=35)$. The total recurrence frequency (local, regional and systemic) was $16.9 \%(n=13)$. The most used adjuvant hormonal treatment was switch therapy $40.3 \%(n=31)$ of the cases. No differences were found in disease-free and overall survival when the biological tumor subtype and the type of adjuvant hormonal treatment received, regarding the safety profile, the treatment administered was quite safe, the most frequent toxicity being arthralgias $11.7 \%(n=9)$ and osteoporosis $10.4 \%(n=8)$.

Conclusions: The median disease-free and overall survival was 30 months and 45.2 months respectively, no differences were documented between the different biological tumor subtypes and the treatment groups, however at the descriptive level it was observed that luminal a tumors as well as patients who received hormonal therapy with switch scheme had a lower recurrence frequency and better overall survival.

Keywords (DeCS): Breast neoplasms, aromatase inhibitors, tamoxifen, mortality, survival.

\section{Introducción}

Para Colombia, según datos de Globocan 2018, el cáncer de mama es el tipo de cáncer más común en las mujeres con 13.380 casos, que representan el 24,8\% de todos los canceres ${ }^{1}$. La mayoría de las pacientes con cáncer de mama en Colombia se diagnostican en etapa localmente avanzada. Piñeros y colaboradores publicaron un estudio descriptivo de las características sociodemográficas, clínicas y de atención de mujeres con cáncer de mama en Bogotá, que el 21,2\% de los cánceres fue temprano (I-IIA); el 57,1\%, localmente avanzado (IIB-IIIC); y el 4,5\%, metastásico (IV)². El cáncer de mama se puede clasificar en distintos subtipos tumorales biológicos, en la duodécima Conferencia Internacional de St. Gallen, Ilevada a cabo en 2011 vigente al momento de la recolección de los datos de nuestro estudio--, se presentó la nueva clasificación que tiene utilidad para estimar el pronóstico de las pacientes con cáncer de mama y orientar el tratamiento sistémico específico para cada uno de los subtipos según la expresión por inmunohistoquímica de los receptores hormonales $(\mathrm{RH})$ de estrógeno y progesterona, la detección de la sobreexpresión o amplificación del receptor HER2 y el Ki67, en tumores luminal A -(receptor de estrógenos o receptor de progesterona positivos, HER2 negativo, Ki67 bajo (<20\%))-. Luminal B HER2 negativo (receptor de estrógenos o receptor de progesterona positivos, HER2 negativo, Ki67 >20\%). Luminal B HER2 positivo (receptor de estrógenos o receptor de progesterona positivos, cualquier Ki67, sobreexpresión o amplificación de HER2), tumores HER2 enriquecidos (HER2 positivo, receptor de estrógenos o receptor de progesterona negativos) y tumores basal-like o triple negativo (receptor de estrógenos o receptor de progesterona negativos, HER2 negativo) $)^{3}$. Para realizar un tratamiento integral de los pacientes con cáncer de mama en estadios localmente avanzados, se debe asegurar un control local y sistémico de la enfermedad a corto y largo plazo, incluyendo el manejo con quimioterapia neoadyuvante y/o adyuvante, procedimientos quirúrgicos para manejo del tumor primario en la mama y axila; terapias blanco, radioterapia y tratamiento hormonal adyuvante para mejorar la supervivencia libre de enfermedad y la supervivencia global ${ }^{4}$. Las pacientes con cáncer de mama $\mathrm{RH}$ positivo deben recibir hormonoterapia adyuvante ya que esta terapia ha demostrado tener impacto sobre la supervivencia libre de recaída tumoral, la supervivencia libre de enfermedad y la global ${ }^{4}$. Tal como se ha podido demostrar en diferentes metaanálisis, como el llevado a cabo en el año 2015, que incluyó 9 ensayos aleatorios ( $n=31,920$ mujeres posmenopáusicas), que comparó el uso de inhibidor de aromatasa (IA) versus tamoxifeno, el cual mostró que cinco años de tratamiento con una IA produce una mejor supervivencia libre de enfermedad y supervivencia global que cinco años de tamoxifeno. En comparación con el tamoxifeno, tomar IA durante cinco años resultó en una disminución de la recurrencia por cáncer de mama, particularmente durante los años 0 a 1 (RR 0,64, IC95\% 0,52-0,78, p<0,001), y durante los años 2 a 4 (RR 0,80, IC95\% 0,68-0,93, p $<0,01)$ sin impacto en las tasas de recurrencia después del período de tratamiento de cinco años. Se presentó menor mortalidad por cáncer de mama a 10 años (RR $0,85, \mathrm{IC} 95 \% 0,75-0,96, \mathrm{p}<0,01)^{5}$. El propósito de este estudio es describir los desenlaces clínicos de supervivencia libre de enfermedad y supervivencia global de pacientes con cáncer de mama localmente avanzado, manejadas con quimioterapia neoadyuvante en el Instituto Nacional de Cancerología (INC) (Bogotá, Colombia), durante los años 2012 a 2014 y que además recibieron dentro de su tratamiento terapia hormonal adyuvante.

\section{Materiales y métodos}

Estudio observacional descriptivo retrospectivo. El estudio incluyó una muestra seleccionada por conveniencia de 109 pacientes con diagnóstico confirmado 


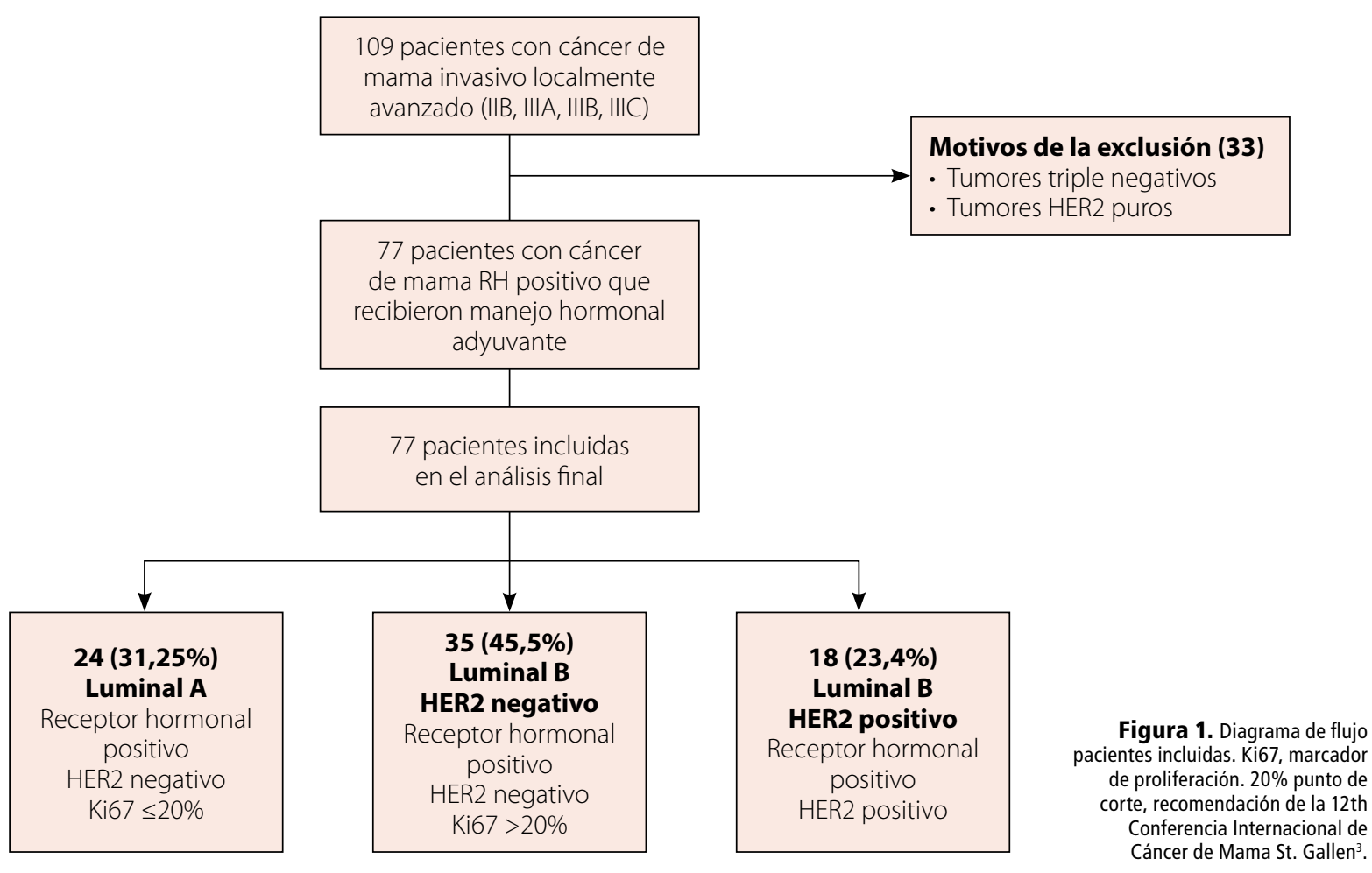

de cáncer de mama invasivo localmente avanzado, que recibieron quimioterapia neoadyuvante durante el período de mayo de 2012 a octubre de 2014. De la muestra total solamente se seleccionaron 77 pacientes con cáncer de mama RH positivo que recibieron manejo hormonal adyuvante. Se excluyeron 33 pacientes por las siguientes razones: 20 (80\%) eran tumores triple negativos y 13 (20\%) eran tumores HER2 puros (figura 1).

Para la recolección de la información sobre las características clínicas e histopatológicas, primero se identificaron las pacientes elegibles mediante revisión de los registros en la historia clínica del sistema SAP en el Instituto Nacional de Cancerología (INC) (Bogotá, Colombia), se corroboró si las pacientes elegibles cumplían los criterios de inclusión y no presentaban algún criterio de exclusión; una vez incluida la paciente en el estudio, se extrae la información y se diligencian las variables propuestas, esto se realiza en un formulario electrónico diseñado para el estudio en la plataforma RedCap, la calidad de la información fue evaluada bajo la supervisión de un monitor de investigación del INC, las variables cualitativas serán presentadas en distribución por frecuencias y porcentajes, y las variables cuantitativas en media y mediana frecuencias, los resultados serán presentados en tablas. Para la estimación de la supervivencia libre de enfermedad y supervivencia global, se usará el método de Kaplan-Meier. Los desenlaces de interés serán supervivencia global, definida como el período de tiempo transcurrido entre la fecha de diagnóstico histológico y la fecha reportada de muerte por cualquier causa; y la supervivencia libre de enfermedad, definida como el tiempo transcurrido entre el tratamiento recibido con intención curativa hasta la recurrencia documentada o la muerte. Para el análisis de la información, se empleará el programa estadístico Stata versión 11.

\section{Resultados}

De los 77 sujetos incluidos, la media de edad fue 53 años con un rango de edad entre 34 y 77 años con una (DE) de 9,8 años, el 61\% $(n=47)$ de las pacientes eran posmenopáusicas. La mayoría de las pacientes fueron clasificadas en estadio clínico IIIB $(51,9 \%, n=40)$ y el tipo histológico tumoral más frecuente fue ductal no especial (NOS) identificado en 71 pacientes (92,2\%), La mediana de tamaño tumoral fue $5,0 \mathrm{~cm}$ con un rango entre $1,5 \mathrm{~cm}$ y $19,5 \mathrm{~cm}$, un total de 40 pacientes (51,9\%) tenían tumores T4b y 50 pacientes (64,9\%) tenían afección ganglionar clínica N1. Los tumores grado II 56 (72,7\%) fueron los más frecuentes, más de la mitad de las pacientes tenían Ki67 mayor del 20\% $50(64,4 \%)$. En cuanto a la expresión de receptores 
hormonales, 59 (76,6\%) de las pacientes tenían expresión mayor del $80 \%$ para receptores de estrógenos y $44(57,1 \%)$ de las pacientes tenían más del $80 \%$ de expresión de receptores de progestágenos. El 89,61\% de las pacientes tenían ambos receptores positivos en la patología (estrógenos y progestágenos) y el 10,38\%

Tabla 1. Características demográficas y clínicas de las pacientes diagnosticadas con cáncer de mama incluidas en el estudio

\begin{tabular}{|c|c|}
\hline Características & $\begin{array}{l}\text { Total de pacientes } \\
(\mathrm{n}=77), \mathrm{n}(\%)\end{array}$ \\
\hline Mediana de edad años & 53 \\
\hline $\begin{array}{l}\text { Género } \\
\text { Femenino }\end{array}$ & $77(100)$ \\
\hline $\begin{array}{l}\text { Estado menopáusico } \\
\text { Menopáusica } \\
\text { Premenopáusica }\end{array}$ & $\begin{array}{l}47(61) \\
30(39)\end{array}$ \\
\hline Mediana de tamaño tumoral en $\mathrm{cm}$ & 5 \\
\hline $\begin{array}{l}\text { Tamaño tumor T } \\
\text { T2 } \\
\text { T3 } \\
\text { T4a } \\
\text { T4b } \\
\text { T4c }\end{array}$ & $\begin{array}{c}22(28,6) \\
12(15,6) \\
2(2,6) \\
40(51,9) \\
1(1,3)\end{array}$ \\
\hline $\begin{array}{l}\text { Compromiso ganglionar } \\
\text { N0 } \\
\text { N1 } \\
\text { N2a } \\
\text { N3a } \\
\text { N3c }\end{array}$ & $\begin{array}{c}5(6,5) \\
50(64,9) \\
18(23,4) \\
1(1,3) \\
3(3,9)\end{array}$ \\
\hline $\begin{array}{l}\text { Estadio clínico } \\
\text { Estadio IIB } \\
\text { Estadio IIIA } \\
\text { Estadio IIIB } \\
\text { Estadio IIIC }\end{array}$ & $\begin{array}{c}20(26) \\
13(16,9) \\
40(51,9) \\
4(5,2)\end{array}$ \\
\hline $\begin{array}{l}\text { Estado funcional } \\
\text { ECOG O } \\
\text { ECOG } 1 \\
\text { ECOG } 2\end{array}$ & $\begin{array}{c}31(40,3) \\
45(58,4) \\
1(1,3)\end{array}$ \\
\hline $\begin{array}{l}\text { Tipo histológico } \\
\text { Ductal (NOS) } \\
\text { Ductal puro } \\
\text { Lobulillar } \\
\text { Mucinoso } \\
\text { Otro }\end{array}$ & $\begin{array}{l}63(81,8) \\
8(10,4) \\
2(2,6) \\
1(1,3) \\
3(3,9)\end{array}$ \\
\hline $\begin{array}{l}\text { Grado tumoral (SBR) } \\
\text { Grado I } \\
\text { Grado II } \\
\text { Grado III }\end{array}$ & $\begin{array}{c}5(6,5) \\
56(72,7) \\
16(20,8)\end{array}$ \\
\hline $\begin{array}{l}\text { Receptores de estrógenos } \\
\text { Negativo } \\
\text { Positivo mayor del } 80 \% \\
\text { Positivo entre } 50 \% \text { y } 79 \% \\
\text { Positivo entre } 49 \% \text { y } 21 \% \\
\text { Positivo menor del } 20 \%\end{array}$ & $\begin{array}{c}5(6,5) \\
59(76,6) \\
3(3,9) \\
4(5,2) \\
6(7,8)\end{array}$ \\
\hline $\begin{array}{l}\text { Receptores de progestágenos } \\
\text { Negativo } \\
\text { Positivo mayor del } 80 \% \\
\text { Positivo entre } 50 \% \text { y } 79 \% \\
\text { Positivo entre } 49 \% \text { y } 21 \% \\
\text { Positivo menor del } 20 \%\end{array}$ & $\begin{array}{c}3(3,9) \\
44(57,1) \\
10(13,0) \\
14(18,2) \\
6(7,8)\end{array}$ \\
\hline $\begin{array}{l}\text { Factor de crecimiento epidérmico humano } 2 \\
\text { Negativo } \\
\text { Equívoco } \\
\text { Positivo }\end{array}$ & $\begin{array}{l}50(64,9) \\
12(15,6) \\
15(19,5)\end{array}$ \\
\hline $\begin{array}{l}\text { FISHODISH } \\
\text { Positivo } \\
\text { Negativo } \\
\text { Sin dato }\end{array}$ & $\begin{array}{c}3(25) \\
8(66,7) \\
1(8,3)\end{array}$ \\
\hline $\begin{array}{l}\text { Ki67 } \\
\text { Menor o igual al 20\% } \\
\text { Mayor del 20\% }\end{array}$ & $\begin{array}{l}27(35,1) \\
50(64,9)\end{array}$ \\
\hline
\end{tabular}

Ki67, marcador de proliferación ce Eastern Cooperative Oncology Group. restante, es decir, 8 pacientes, solo tenía positivo uno de los dos receptores hormonales (tabla 1). Con respecto a la clasificación por subtipos moleculares de cáncer de mama, los tumores luminales A fueron $31,2 \%$ de los casos con 24 pacientes; luminal B HER2 negativo se describió en 35 (45,5\%); luminal B HER2 positivo en $18(23,3 \%)$ (figura 1).

El tipo de tratamiento hormonal adyuvante más utilizado fue la terapia switch en $40,3 \%(n=31)$ de los casos y de estas el esquema de tamoxifeno-anastrozol fue el más común en el $26 \%(n=20)$ de los casos, seguida de la terapia inicial con inhibidor de aromatasa en el $18(23,45 \%)$; y $11(14,3 \%)$ de los pacientes terapia inicial con tamoxifeno (tabla 2).

Durante un tiempo total de seguimiento de 5 años, la frecuencia total de recurrencia (local, regional y sistémica) fue del $16,9 \%(n=13)$, de los cuales 2 casos $(15,4 \%)$ fueron locales a tejidos blandos y 11 a distancia $(84,6 \%)$, los sitios afectados con mayor frecuencia por metástasis fueron hueso $8(61,5 \%)$; ganglios no regionales 7 (53,8\%); pulmón 5 (38,5\%) e hígado 4 $(30,8 \%)$. Con respecto a los dos casos que presentaron recaída local a tejidos blandos, fueron manejados con cirugía + hormonoterapia. El tratamiento más utilizado para la recaída fue quimioterapia $30,8 \%(n=4)$ seguido de quimioterapia + radioterapia $15,4 \%(n=2)$ y cirugía + hormonoterapia $15,4 \%(n=2)$, se presentó un caso de recaída sistémica que no recibió tratamiento por

Tabla 2. Frecuencias absolutas y relativas por tipo de tratamiento de pacientes diagnosticadas con cáncer de mama

\begin{tabular}{|c|c|}
\hline Tratamiento & $\begin{array}{l}\text { Total, de pacientes } \\
\qquad(\mathrm{n}=77), \mathrm{n}(\%)\end{array}$ \\
\hline $\begin{array}{l}\text { Régimen de quimioterapia neoadyuvante } \\
\text { AC-T } \\
\text { AC-TH } \\
\text { AC solo } \\
\text { TC solo } \\
\text { Otros (paclitaxel + trastuzumab) }\end{array}$ & $\begin{array}{l}56(72,7) \\
17(22,1) \\
2(2,6) \\
1(1,3) \\
1(1,3)\end{array}$ \\
\hline $\begin{array}{l}\text { Tiempo de la quimioterapia neoadyuvante } \\
\text { Menor de } 4 \text { meses } \\
\text { Entre } 4 \text { y } 6 \text { meses } \\
\text { Más de } 6 \text { meses }\end{array}$ & $\begin{array}{c}1(1,3) \\
55(71,4) \\
21(27,3)\end{array}$ \\
\hline $\begin{array}{l}\text { Cirugía del tumor primario } \\
\text { Cuadrantectomía + vaciamiento axilar } \\
\text { Mastectomía radical modificada }\end{array}$ & $\begin{array}{l}24(31,2) \\
52(67,5)\end{array}$ \\
\hline $\begin{array}{l}\text { Tipo de hormonoterapia adyuvante } \\
\text { Inicial con tamoxifeno } \\
\text { Inicial con inhibidor de aromatasa } \\
\text { Análogo de LHRH + tamoxifeno } \\
\text { Switch tamoxifeno-letrozol } \\
\text { Switch tamoxifeno-anastrozol } \\
\text { Switch tamoxifeno-exemestano } \\
\text { Hormonoterapia extendida con tamoxifeno } \\
\text { Hormonoterapia extendida con inhibidor de aromatasa }\end{array}$ & $\begin{array}{c}11(14,3) \\
18(23,4) \\
1(1,3) \\
9(11,7) \\
20(26) \\
2(2,6) \\
5(6,5) \\
11(14,3)\end{array}$ \\
\hline $\begin{array}{l}\text { AC: adriamicina + ciclofosfamida } \\
\text { AC-T: adriamicina + ciclofosfamida-taxano } \\
\text { AC-TH: adriamicina+ ciclofosfamida-taxano + trastuzumab } \\
\text { AC-TC: adriamicina + ciclofosfamida-taxano + carboplatino } \\
\text { TC: taxano + carboplatino }\end{array}$ & \\
\hline
\end{tabular}


deterioro clínico y posterior fallecimiento en el 7,7\%. Al relacionar la supervivencia libre de enfermedad de las pacientes de la cohorte con algunas variables clínicas, se encontró que con respecto al estadio clínico las pacientes que presentaron mayor recaída fueron estadio IIIB 69,2\% ( $n=9)$, con relación al subtipo molecular 9 $(69,2 \%)$ eran luminal B HER2 negativo, los tumores con grado II representaban el $84,6 \%(n=11)$ de las recaídas y $92,3 \%$ de las recaídas eran tumores con índice de proliferación Ki67 mayor del 20\%.

La mediana del tiempo de seguimiento fue de 4,6 años, el seguimiento fue definido con corte a julio de 2018. En la figura 2 se puede ver el comportamiento a nivel general de la supervivencia global y libre de recaída. Se puede ver que la supervivencia global a 30 meses fue mayor con respecto a la supervivencia libre de recaída, con 0,9859 y 0,9019, también a 60 meses se evidenciaron supervivencias de 0,9348 y 0,7529 respectivamente.

Se registraron un total de 4 muertes secundarias a enfermedad, lo que representa el 5,2\% del total de pacientes, de las cuales el $100 \%(n=4)$ era estadio IIIB, respecto al subtipo molecular el $75 \%(n=3)$ eran luminal B HER2 negativo y el $25 \%(n=1)$ eran luminal B HER2 positivo, los tumores grado III representaban el 50\% $(n=2)$ de las muertes y el $100 \%$ de estas eran tumores con índice de proliferación Ki67 mayor del 20\%. En la figura 3 se presenta el comportamiento de las curvas de supervivencia global de acuerdo con algunas variables de tipo clínico, biológica y de tratamiento de hormonoterapia adyuvante -considerando los tratamientos hormonoterapia extendida con inhibidor de aromatasa (HEIA), inicial con inhibidor de aromatasa (IIA), inicial con tamoxifeno (IT) y switch tamoxifeno letrozol (STL), dado que en estos es donde se encontraron los eventos de interés: recaída o fallecimiento)-. Debido al pequeño número de eventos registrados no se documentaron diferencias entre los estadios clínicos, subtipos moleculares y tipo de tratamiento hormonal adyuvante recibido, pero a nivel descriptivo las pacientes con estadios IIIB y IIIC y los subtipos luminal B HER2 negativo y luminal

Tabla 3. Caracterización de la toxicidad en términos de frecuencias absolutas y relativas de pacientes diagnosticadas con cáncer de mama

\begin{tabular}{|l|c|}
\hline \multicolumn{1}{|c|}{ Toxicidad reportada } & $\begin{array}{c}\text { Total, de pacientes } \\
(\mathbf{n = 7 7}), \mathbf{n}(\%)\end{array}$ \\
\hline Sí & $20(26)$ \\
No & $53(68,8)$ \\
Sin dato & $4(5,2)$ \\
\hline Tipo de toxicidad & \\
Artralgias & $9(11,7)$ \\
Mialgias & $7(9,1)$ \\
Dislipidemia & $3(3,9)$ \\
Osteoporosis & $8(10,4)$ \\
Elevación de transaminasas & $1(1.3)$ \\
\hline Interrupción del tratamiento & \\
Sí & $15(19,5)$ \\
No & $58(75,3)$ \\
Sin dato & $4(5,2)$ \\
\hline Problemas administrativos dela EPS & $6(40,0)$ \\
Intolerancia al medicamento & $1(6,7)$ \\
Progresión de la enfermedad & $5(33,3)$ \\
Otros & $3(20,0)$ \\
\hline Número de controles programados (años) & \\
5 & $77(100)$ \\
\hline Número de controles realizados (años) & \\
0 & $1(1,3)$ \\
1-2 & $9(11,7)$ \\
3-4 & $11(14,3)$ \\
5 & $56(72,7)$ \\
\hline Asistió a todos los controles & \\
Sí & $56(76,6)$ \\
No & $18(23,4)$ \\
\hline
\end{tabular}
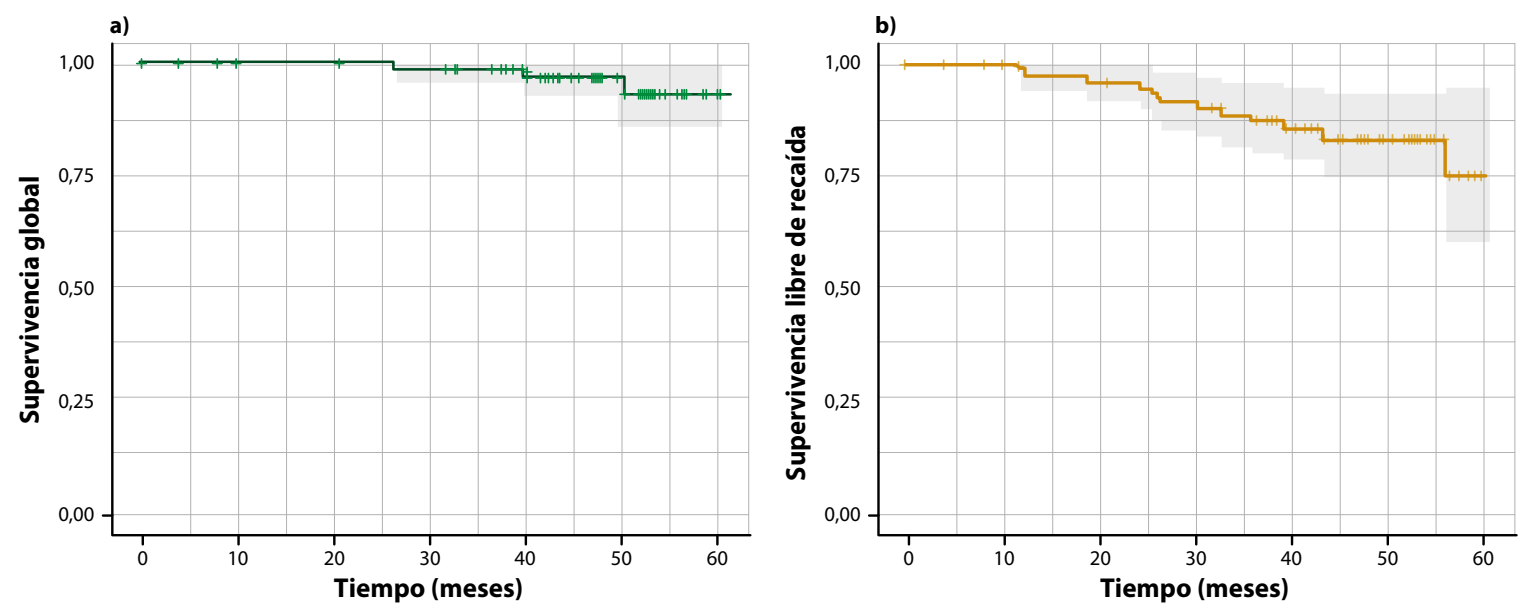

Figura 2. Curvas de supervivencia por método de Kaplan-Meier. a) Global. b) Libre de recaída, en pacientes diagnosticadas con cáncer de mama. 


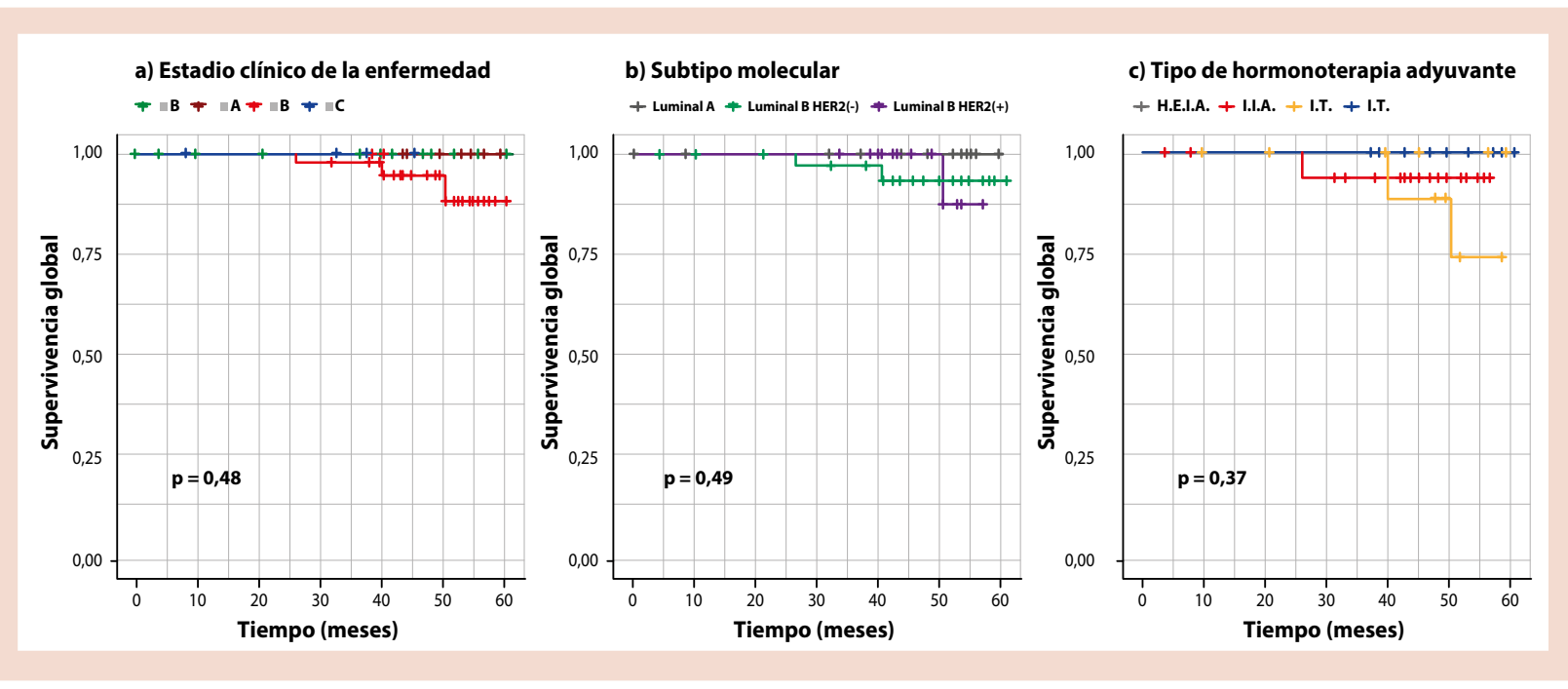

Figura 3. Curvas de supervivencia global en pacientes diagnosticadas con cáncer de mama, de acuerdo con algunas variables clínicas y de tratamiento4, estimador de Kaplan-Meier. ${ }^{4}$ Abreviatura para tratamientos considerados en el análisis: hormonoterapia extendida con inhibidor de aromatasa (HEIA), inicial con inhibidor de aromatasa, inicial con tamoxifeno (IT) y switch tamoxifeno letrozol (STL).

B HER2 positivo, así como las pacientes que recibieron tratamiento inicial solo con tamoxifeno, tenían menores resultados en supervivencia global cuando se comparan con tumores en estadios IIB y IIIA y tumores luminal A y tratamiento con terapia switch o inicial con inhibidor de aromatasa, respectivamente (figura 3).

En cuanto a la seguridad y continuidad de la terapia hormonal adyuvante, en el $68,8 \%(n=53)$ no se reportó toxicidad con adecuada tolerancia y adherencia, en solo el $26 \%(n=20)$ de los casos se reportó toxicidad, principalmente $9(11,7 \%)$ casos de artralgias, 8 (10,4\%) de osteoporosis, 7 (9,1\%) de mialgias, 3 de dislipidemia y 1 (1,3\%) de elevación de transaminasas (tabla 3). El 73,3\% ( $n=58)$ no presentó interrupción del tratamiento, mientras que en el $19,5 \%(n=15)$ sí se presentó, siendo la principal causa de interrupción en un $40 \%(n=6)$ problemas administrativos por parte de la EPS y $5(33,3 \%)$ casos por progresión de la enfermedad (tabla 3).

\section{Discusión}

En Colombia el 53,9\% de las pacientes con cáncer de mama que ingresan al Instituto Nacional de Cancerología se encuentran en etapa localmente avanzada de la enfermedad, según el reporte del Anuario estadístico 2015 publicado por el INC6. El pronóstico de los pacientes con enfermedad localmente avanzada es relativamente más pobre cuando se compara con estadios más tempranos de la enfermedad, por ejemplo, la tasa de supervivencia relativa a cinco años para enfermedad en estadio I es de $95 \%$ y de solo $48 \%$ para tumores estadio $\mathrm{IIB}^{7}$. Por otro lado, la biología tumoral más frecuente a nivel global son tumores luminales (alrededor del 70\%)3. Conforme con el estudio Precama, coordinado por la Agencia Internacional para la Investigación del Cáncer, realizado con mujeres premenopáusicas de cuatro países de América Latina (Chile, Colombia, Costa Rica y México), se evidenció que el $72 \%$ de los tumores eran receptor hormonal positivo, el $19 \%$ fueron HER2 positivos y el $21 \%$ fueron triples negativos ${ }^{8}$. Otro estudio efectuado a nivel local, con 114 participantes, demostró que en Colombia el subtipo luminal A es el más frecuente, en el $38,5 \%$ de tumores, seguidos de luminal $B(32,4 \%)$, triple negativo $(15,8 \%)$ y HER2 positivo, con el $13,1 \%{ }^{9}$. Por lo que la población seleccionada en este estudio es un reflejo importante de las mujeres con esta enfermedad; además, este estudio fue llevado a cabo en un centro de referencia para el tratamiento del cáncer a nivel nacional y regional para Latinoamérica.

El cáncer de mama es una enfermedad altamente heterogénea con respecto a sus características clínicas y comportamiento biológico. El conocimiento actual de los subtipos moleculares ha proporcionado un mejor enfoque para su tratamiento ${ }^{10}$. Los tumores luminales están asociados a bajo riesgo de recurrencia local y regional y un pronóstico altamente favorable ${ }^{11}$. En términos de tasa de recurrencia local, varios estudios retrospectivos han mostrado resultados similares con porcentajes que oscilan entre el $0,8 \%$ y $8 \%$ para 
tumores luminal $A$; mientras que el subtipo molecular luminal $B$ está asociado a un pronóstico más intermedio en comparación con el subtipo molecular luminal A; el riesgo de recurrencia local, como se describe en la literatura para tumores luminal $B$, varía entre el 1,5\% y $8,7 \%$ con un pico de incidencia en los primeros cinco años después del diagnóstico ${ }^{12}$.

En nuestro estudio no se documentó ningún caso de recurrencia local en tumores luminal $A$, mientras que se reportó $1(7,7 \%)$ caso de recurrencia local en un tumor subtipo molecular luminal B HER2 negativo y otro caso $(7,7 \%)$ en un tumor luminal B HER2 positivo ,información similar a la reportada en la literatura global sin observar diferencias en frecuencia de recurrencia local en los subtipos luminal B (HER2 positivo y negativo) en nuestro análisis, sobre todo teniendo en cuenta que el $100 \%(n=18)$ de los tumores luminal B HER2 positivo en nuestro estudio recibieron tratamiento con trastuzumab. A nivel descriptivo, la supervivencia global y libre de recaída fue menor en luminal B HER2 (+) y luminal B HER2 (-), respectivamente (figura 3). Cuando se analiza la localización de la recaída según el subtipo tumoral biológico, los tumores luminales tienen más probabilidades de desarrollar metástasis clínicamente aparentes a hueso, tejidos blandos y sistema reproductor femenino, mientras que los tumores RH negativos con mayor frecuencia hacen metástasis a sistema nervioso central, hígado, sitios que están asociados con una supervivencia más corta13. En nuestro análisis se documentaron 13 recaídas que correspondían a un $16,9 \%$ del total de la muestra y de estas el $61,5 \%(n=8)$ de los casos presentaron metástasis a hueso y el 53,8\% $(n=7)$ de los casos a ganglios linfáticos no regionales, datos similares a los descritos en la literatura, pues se trata de tumores luminales.

En cuanto a la terapia endocrina adyuvante, está recomendada a todas las pacientes con tumores luminales, independiente de la edad y del riesgo de recurrencia ${ }^{14}$. Medicamentos como el tamoxifeno reducen el riesgo de recurrencia durante los años $0-4$ en casi un $50 \%$ con una disminución continua del riesgo de más del 30\% durante los años 5-9. Además, la mortalidad anual por cáncer de mama decrece en un 30\% durante los primeros 15 años $^{15}$. Otra clase de medicamentos como los inhibidores de aromatasa (IA) han podido demostrar beneficios en comparación con tamoxifeno, tal como se demostró en el metaanálisis del grupo EBCTCG en el año 2015, donde se evidenció una reducción de riesgo de recurrencia del cáncer en un 30\%, y en el riesgo de morir por cáncer de mama en alrededor del 15\% durante la primera década ${ }^{5}$. En nuestro estudio el 40,3\% ( $\left.n=31\right)$ de las pacientes recibieron terapia hormonal adyuvante switch con tamoxifeno, seguida de un IA y, de estos, el esquema más utilizado fue tamoxifeno-anastrozol en un $26 \%$ ( $n=20)$, elección que puede estar condicionada por las características que confieren riesgo de recurrencia en nuestra población, pues se trataba de lesiones con gran tamaño tumoral (mediana de tamaño $5 \mathrm{~cm}$ ) y compromiso ganglionar en un $64,9 \%(n=50)$ cN1 y tumores grado II SBR en un $72,7 \%(n=56)$. El segundo esquema de manejo hormonal adyuvante que se administró con más frecuencia fue terapia inicial con IA en un $23,4 \%$ ( $n=18)$ de los casos que puede ser explicado por las características de riesgo previamente descritas y porque la gran mayoría de mujeres en nuestro análisis se encontraban en estado de menopausia al momento del inicio de la terapia $61 \%(n=47)$; en cuanto a los desenlaces de supervivencia libre de enfermedad y supervivencia global, no se documentaron diferencias entre los diferentes grupos de tratamiento, sin embargo, a nivel descriptivo el grupo de terapia inicial con tamoxifeno presentó menores resultados (figura 3).

Con relación a la seguridad y adherencia al tratamiento hormonal adyuvante, en términos generales es bien tolerado ${ }^{16}$. Dentro de los efectos secundarios asociados con el uso de IA, está el desarrollo de osteoporosis acelerada, por lo que las pacientes que los reciben requieren la monitorización seriada de la densidad mineral ósea ${ }^{17}$. En nuestro análisis se registró un $26 \%$ $(n=20)$ de casos de toxicidad, siendo la más frecuente aparición de artralgias 45\% ( $n=9)$; el segundo evento que se presentó con más frecuencia fue la manifestación de osteoporosis en un $40 \%(n=8)$ de los casos, sin que se registrara un evento de fractura clínica, datos similares a los descritos en la literatura mundial sin que esta toxicidad pudiese condicionar interrupción del tratamiento, pues solamente se registró 1 caso $(6,7 \%)$ de interrupción de este tratamiento debido a intolerancia con el manejo hormonal adyuvante, reflejo de la buena adherencia y seguridad que presentó nuestra cohorte; no obstante, se debe tener en cuenta que existen múltiples factores relacionados con la adherencia terapéutica del paciente al tratamiento. Dentro de estos se encuentran los relacionados con las organizaciones 
prestadoras de salud. En el estudio de Suárez, se concluye que las principales causas de abandono detectadas se relacionaron con el sistema de seguridad social. De los pacientes objeto de este estudio, el 38,44\% $(n=15)$ aseguraron haber abandonado el tratamiento por dificultades con las autorizaciones de servicios y medicamentos ${ }^{18}$. En nuestro estudio se documentó que el $40 \%(n=6)$ de los casos presentó interrupción del tratamiento por problemas administrativos, información similar a la descrita a nivel local. Por lo demás la adherencia fue buena, ya que el 76,6\% ( $n=56)$ asistió a todos los controles programados (tabla 3 ).

El estudio muestra el comportamiento de la enfermedad localmente avanzada, forma de presentación más frecuente en nuestro medio, pues se aprecia el patrón de uso de la manipulación hormonal, sugiriendo a nivel descriptivo que la terapia upfront con tamoxifeno y las pacientes con subtipo biológico tumoral luminal B tienen peores resultados, por lo que nuestros hallazgos reflejan la necesidad de realizar un diagnóstico más temprano y brindar un tratamiento más oportuno.

\section{Conclusiones}

La mediana de supervivencia libre de enfermedad y global fue de 30,07 y 45,23 meses, respectivamente; no se describen diferencias entre los subtipos tumorales biológicos y los grupos de tratamiento, sin embargo, a nivel descriptivo en este análisis se observó que los tumores luminal $A$, al igual que los pacientes que recibieron terapia de manipulación hormonal con esquema switch, tenían mejor pronóstico, una menor frecuencia de recurrencia y mayor supervivencia global cuando se comparan con tumores luminal B y pacientes que reciben terapia inicial con tamoxifeno.

\section{Referencias}

1. Bray F, Ferlay J, Soerjomataram I, et at. Global cancer statistics 2018: Globocan estimates of incidence and mortality worldwide for 36 cancers in 185 countries. CA Cancer J Clin. 2018; 68:394-424.

2. Piñeros $M$, Sánchez $R$, Cendales $R$, et al. Características sociodemográficas, clínicas y de la atención de mujeres con cáncer de mama en Bogotá. Revista Colombiana de Cancerología. 2008;12(4):181-90

3. Vasconcelos I, Hussainzada A, Berger S, et al. The St. Gallen surrogate classification for breast cancer subtypes successfully predicts tumor presenting features, nodal involvement, recurrence patterns and disease free survival. The Breast. 2016;29:181-5

4. Moo TA, Sanford R, Dang Ch, et al. Overview of breast cancer therapy. PET Clinics. 2018;13(3):339-54.

5. Early Breast Cancer Trialists' Collaborative Group (EBCTCG). Aromatase inhibitors versus tamoxifen in early breast cancer: patient-level meta-analysis of the randomised trials. Lancet. 2015;1341-52.

6. Instituto Nacional de Cancerología-ESE. Anuario estadístico 2015. Colombia: Instituto Nacional de Cancerología-ESE; 2018. Disponible en: https://www.cancer.gov.co/files/libros/ archivos/2015

7. Newman LA, Epidemiology of locally advanced breast cancer. Semin Radiat Oncol. 2019;19:195-203.

8. Romieu I, Biessy C, Carayol M, et al. Reproductive factors and molecular subtypes of breast cancer among premenopausal women in Latin America: The Precama study. Sci Rep. 2018; 8:13109.

9. Bonilla-Sepúlveda O, Matute-Turizo G, Severiche C. Clasificación en subtipos intrínsecos de los carcinomas de mama analizados en un centro de patología de Medellín en el año 2011. CES Medicina. 2015;29:12.
10. Puig-Vives $M$, Sánchez MJ, Sánchez-Cantalejo J. Distribution and prognosis of molecular breast cancer subtypes defined by immunohistochemical biomarkers in a spanish population-based study. Gynecologic Oncology. 2013;130:609-14.

11. Fragomeni SM, Sciallis $A$, Jeruss JS. Molecular subtypes and local-regional control of breast cancer. Surg Oncol Clin N Am. 2018;27(1):95-120.

12. Millar EKA, Graham PH, O'Toole SA. Prediction of local recurrence, distant metastases, and death after breast-conserving therapy in early-stage invasive breast cancer using a five-biomarker panel. J Clin Oncol. 2009;27:4701-8.

13. Insa A, Lluch A, Prosper F. Prognostic factors predicting survival from first recurrence in patients with metastatic breast cancer: analysis of 439 patients. Breast Cancer Research and Treatment. 1999:56:67-78

14. NCCN clinical practice guidelines in Oncology (NCCN Guidelines $($ ) breast cancer version 1.2019 - march 14, 2019.

15. Early Breast Cancer Trialists' Collaborative Group (EBCTCG). Relevance of breast cancer hormone receptors and other factors to the efficacy of adjuvant tamoxifen: patient-level metaanalysis of randomised trials. Lancet. 2011;378:771-84.

16. Crew KD, Greenlee H, Capodice J, et al. Prevalence of joint symptoms in postmenopausal women taking aromatase inhibitors for early-stage breast cancer. J Clin Oncol. 2007;25(25):3877-83

17. Van Poznak C, Somerfield MR, Moy B, et al. Role of bone-modifying agents in metastatic breast cancer: an American Society of Clinical Oncology-Cancer Care Ontario focused guideline update. J Clin Oncol. 2017;35(35):3978-86.

18. Suárez A, Guzmán C, Villa B, Gamboa O. Principales causas de abandono de tratamiento: una causa de muerte evitable en el niño con cáncer. Revista Colombiana de Cancerología. 2011. 